\title{
Vulnerability Analysis of a Nuclear Power Plant Considering Detonations of Explosive Devices
}

\author{
Marko ČEPIN*, Leon CIZELJ, Matjaž LESKOVAR, Borut MAVKO \\ Jožef Stefan Institute, Ljubljana 1000, Slovenia \\ marko.cepin@ijs.si
}

\begin{abstract}
The needs for vulnerability analyses picked up the pace after the military threats to a nuclear power plant in the year 1991 and after the 9/11 events in 2001. The methodology which was proposed for complex assessment of possible consequences following a deliberate damage, shortly after the year 1991 is here further developed to correspond to requests for further studies identified after the events $9 / 11$. The new methodology integrates phenomenological models of the cause of damage, material strength and injuries of human beings with nuclear power plant models used in probabilistic safety assessment. The damage source studied is an explosion of a device brought to the location by land transport. The description of the method and its results are only illustrative and not very detailed in order that the results can not be used for malicious purposes. A straightforward example analyzing the response of a simplified process facility to a ground explosion outside the building is shown, although the methodology was tested also on a power plant. The results indicate that sizable explosions are required to inflict any damage to the reinforced concrete walls. Much larger explosions are needed to break the equipment behind such walls. The performed analysis shows that the facility can be even better secured at relatively low costs.
\end{abstract}

Key words: vulnerability, explosion, blast, probabilistic safety assessment, nuclear.

\footnotetext{
${ }^{*}$ Corresponding author, Tel. +386-1-588-5263, Fax. +386-1-588-5377, E-mail: marko.cepin@ijs.si ${ }^{\dagger}$ Present address: Institut Jožef Stefan, Jamova 39, 1000 Ljubljana, Slovenia
} 


\section{Introduction}

The first comprehensive analysis of nuclear power plant (NPP) vulnerability to a deliberately caused damage goes back to the year 1991, when an operating NPP was exposed to a military threat ${ }^{1)}$. Fortunately, there was no damage and the threat ceased very soon after it was placed. Only a limited international attention was therefore devoted to this event.

Later, intentional damage received much more attention, especially after the tragic 9/11 events: see for example ${ }^{2), 3), 4)}$. Examples studied primarily selected events and their immediate impact on selected NPP systems, such as for example assessment of the intentional damage to the nuclear power plant containment buildings through aircraft impacts ${ }^{5)}$. The results available indicate that NPPs are rather difficult targets ${ }^{2)}$, 4), 5). Such results are to some extent expected, since a lot of efforts have been devoted to the defense in depth, resulting in a reliable and fault tolerant design of the current NPP fleet. More efforts are however needed to improve both the understanding of possible sources of damage and their consequences on the highly complex systems of the NPP.

In this paper, the methodology aiming at complex assessment of possible consequences following a deliberate damage proposed in ${ }^{1)}$ is further developed. It integrates phenomenological models of the cause of damage, material strength and injuries of human beings with the available NPP models used in probabilistic safety assessment. The main intention was to develop a quick and robust assessment method, which to a large extent relies on the knowledge, models and analyses, which already exist. For these reasons, the existing framework of the external event analysis in PSA ${ }^{10}$ ) seemed to provide an excellent starting point.

The particular damage source studied in this paper is an explosion of a device brought to the location by land transport ${ }^{6)}$, 7), 8). A broad sensitivity study varying the amount and position of the explosive has been conducted to assess the vulnerability of the plant and to set up the basis for an effective protection, if the results would show it is needed.

The description of the method and its results in the paper are illustrative and not very detailed in order that the results can not be used for malicious purposes. 
Quantitative values of the selected parameters are not shown to scale neither in figures nor in the text. A simple example analyzing a response of a simplified technological facility to a ground explosion outside the building is shown in the paper, although the analysis was performed for a nuclear power plant, in addition. A discussion of efficient countermeasures based on the assessment results is given.

\section{Method}

The methodology proposed in this paper may well fall into the framework for the analysis of external events, which is routinely carried out in probabilistic safety assessment ${ }^{10), 11)}$. The main analysis steps to be developed and carried out are therefore known in the external events analysis ${ }^{9), 10)}$ and are categorized as:

- hazard analysis,

- evaluation of component fragility and vulnerability,

- plant systems and sequence analysis,

- consequence analysis.

For the selected damage source (explosion of a device brought to the location by land transport), the following main analysis steps were developed and adapted to suit the purpose of the vulnerability analysis of a NPP:

- hazard analysis:

- Blast Analysis, describing the intensity of an explosion in terms, suitable for assessment of damage on structures, systems and personnel.

- component fragility and vulnerability:

- Structural Response to Blast Loading, associating the intensity of explosion from the Blast Analysis with potential damage to the plant buildings and structures.

- Components and Systems Fragility Analysis, associating the intensity of explosion from the Blast Analysis and potential structural damage from the Structural Response to Blast Loading with potential damage to the vital plant systems and/or their components. 
- Human Response to Blast Loading, associating the intensity of explosion from the Blast Analysis with potential damage to the plant personnel.

- plant systems and sequence analysis,

- Plant Damage Analysis, which represents the assessment of the plant damage due to selected explosions.

- Plant Response Analysis, which represents the plant response with probabilistic safety assessment models, which are reevaluated considering the plant damage analysis.

- consequence analysis.

- Consequences analysis, which covers the environmental aspects of ground explosions.

\section{Blast analysis}

An explosion is a very fast chemical reaction producing transient air pressure waves called blast waves ${ }^{12), 13)}$. For a ground-level explosive device such as a bomb in a vehicle, the pressure wave will travel away from the source in the form of a hemispherical wavefront if there are no obstructions in the path. Important parameters of the pressure wave: the peak overpressure and the duration of the overpressure, vary with distance from the device. The magnitude of these parameters depends also on the explosive materials from which the bomb is made and the packaging method of the bomb. Usually the size of the bomb is given in terms of the weight of an equivalent bomb made from trinitrotoluene $(\mathrm{TNT})^{12), 13)}$.

The distance between the bomb and the target building, i.e. stand-off distance, is a fundamental parameter when determining the blast pressures experienced by a building. As stand-off distance increases, blast pressure drops significantly. Therefore, putting distance between the building and the bomb is extremely helpful in reducing blast effects on the building.

\section{Structural Response to Blast Loading}

The buildings in a typical NPP with Pressurized Water Reactor (PWR), which are important for the technological processes and plant safety, may be divided in two broad categories: 
- Reinforced concrete buildings with walls made of reinforced concrete.

- Steel frame buildings with metal sheet walls.

Each of these categories exhibits principally different behavior under blast loadings.

\section{(a) Reinforced concrete buildings}

The first natural frequencies of the buildings are typically below $10 \mathrm{~Hz}$. This leads to the natural periods exceeding $100 \mathrm{~ms}$. For the closely located explosive masses considered, the duration of the overpressure phase is in the order of $10 \mathrm{~ms}$. It is therefore decided to treat the blast load as an impulse load (e.g., the impulse of the reflected overpressure is converted into kinetic energy, which is in turn equilibrated by the strain energy of the building).

The load carrying capacity of the reinforced concrete building is therefore characterized by its elastic strain energy. It is further assumed that the behavior of the building may be reasonably well predicted from the behavior of a set of walls. These in turn are modeled using available elastic solutions for (clamped) plates assuming homogenous behavior of "concrete and steel". The plastic or irreversible deformation energy is accounted for using the ductility ratio ${ }^{14)}$ between the actual deformation energy stored in the wall and the maximum elastic deformation energy. The load carrying capacity of the wall in terms of the impulse caused by the reflected blast overpressure $I_{r}$ is therefore proportional to:

$$
I_{r} \propto h m \sigma_{Y} \sqrt{\frac{(v-1) \rho}{E(1+v)(2 v-1)}},
$$

where $h$ represents the plate thickness, $m$ the ductility ratio, $\sigma_{Y}$ the yield strength, $\rho$ the density, $E$ the Young's modulus and $v$ the Poisson's ratio. Given the particular choice of material, the load carrying capacity is simply governed by the plate thickness $h$. Although not shown in the above equation, the proportionality constant also depends on the aspect ratio of the plate width and the height and results in the highest load carrying capacity of a square plate. The aspect ratio of 2 is therefore conservatively used in further calculations.

Structural response of buildings was divided into three different levels of damage: 
- "no damage" - there is no permanent deformation/damage to the reinforced concrete plate - purely elastic response with $m=1$;

- "wall collapse" occurs when the strain energy in the plate exceeds 20-times the "no damage" strain energy $(m \geq 20)$;

- "destroy systems" and/or structures in the first compartment immediately after the collapsed wall $(m \geq 40)$.

Appropriate correlations were developed to associate the damage levels of the walls with the mass and stand-off distances of explosive charges ${ }^{19), 20), 21), 22), 23) .}$

\section{(b) Steel frame buildings with metal sheet walls}

The load carrying capacity of steel frame buildings with metal sheet walls was assessed using the vulnerability data for "light frame factories" as published in ${ }^{12)}$. Three damage levels were defined consistently with the damage levels defined in section II.2.(a).

Appropriate correlations were developed to associate the damage levels of the walls with the mass and stand-off distances of explosive charges 19$), 20), 21), 22), 23$ ).

\section{Components and Systems Fragility Analysis}

The components and systems fragility analysis takes place only in buildings or behind the walls with damage levels "wall collapse" and/or "destroy systems", see section II.2.(a). For the walls and/or buildings with damage level "wall collapse", immediate failure was assumed for the equipment:

- which is mounted on the collapsed wall;

- which proper operation depends on the air pressure or on change of air pressure in its vicinity.

For the walls and/or buildings with damage level "destroy systems", immediate failure was assumed for all equipment in the first compartment behind the damaged wall.

\section{Human Response to Blast Loading}

Human response to blast loading may be assessed considering three identified categories of blast-induced injury ${ }^{12), 13)}$. These categories are: 
- Primary injury directly due to blast wave overpressure and duration. The location of most severe injuries is where the density differences between adjacent body tissues are greatest.

- Secondary injury due to impact by missiles, e.g. fragments from bomb casing and surrounding material.

- Tertiary injury due to displacement of the entire body, which will inevitably be followed by high decelerative impact loading when most damage occurs.

Among the most descriptive measures for assessing the human response, the damage distances for the occurrence of the primary injuries: eardrum rupture and lung damage death, are identified. In the method both, the eardrum rupture and the lung damage death, are considered and assessed ${ }^{6}$.

\section{Plant Damage Analysis}

The first step for the plant damage analysis is to identify:

- plant buildings, their location and their blast response properties,

- systems important for safety and their location.

Then, the levels of the analysis are identified. The levels of the analysis considering the location and mass of the explosive are the following (usually 3 levels, but in case of more fences within technological buildings the number of levels might be increased accordingly):

- Explosion outside the controlled area (outside of the fence).

- Explosion inside the controlled area (inside of the fence), but outside of the area with technological buildings.

- Explosion inside the area with technological buildings. This level is not explicitly treated here since it is applicable mostly for facility specific analyses. It should however be noted that the analysis of this level would closely follow the analysis inside the control area.

And, finally, the analysis is performed for each level considering the selected explosive masses in three steps: 
- Determination of iso-damage contour lines. An iso-damage line is defined as a line, which for a given mass and location of the explosive charge delineates the areas with different damage levels of the buildings.

- Determination of system failure contour lines. A system failure contour line is defined as a line, which for a given mass and location of the explosive charge delineates the areas with assumed damage to the plant systems and components.

- Determination of critical zones. A critical zone is defined as an area, where a detonation of a charge with a given mass could lead to the damage of the plant systems/components.

\section{(a) Determination of iso-damage contour lines}

Iso-damage contour lines for structural damage and human injuries are determined using damage distances. Iso-damage contour lines are lines connecting explosion locations, which would cause the same specified damage to structures or the same specified injuries to human beings. If the location of the bomb is on the outer side of the iso-damage contour line (the distance between the bomb and the building is greater) the damage would be smaller than the specified damage, and if the location of the bomb is on the inner side of the iso-damage contour line (the distance between the bomb and the building is smaller) the damage would be greater than the specified damage. The iso-damage contour lines are used to establish regions of explosion locations causing a specified damage to structures or causing a specified injury to human beings.

It is conservatively assumed that between the bomb location and the plant buildings there are no obstacles, which would weaken the blast.

\section{(b) Determination of system failure contour lines}

System (component) failure contour lines are determined using damage distances. System failure contour lines are lines connecting the most distant explosion locations, which would still cause the failure of the considered system or component. If the location of the bomb is on the inner side of the system failure contour line (the distance between the bomb and the building is smaller) the system would fail, and if the location of the bomb is on the outer side of the system failure contour line (the distance 
between the bomb and the building is greater) the system would remain intact. The system contour lines are used to establish regions of explosion locations causing a system or component failure.

It is conservatively assumed that all systems in the proximity of the explosion will fail, and not only the systems, which are in the first compartment immediately after the collapsed wall. The reason for such an approach is that due to the complex branched systems piping and electricity installation it is difficult to determine where the installation is actually placed and when it will actually fail. For some systems (components) it was assumed due to their specifics that they are destroyed already when the condition for the wall collapse is fulfilled (see section II.3).

\section{(c) Determination of critical zones}

Critical zones are determined using system failure contour lines. Critical zones are zones around the main model plant buildings, where the explosion would destroy several systems (components) simultaneously, e.g. redundant systems, or more trains of a safety system, or systems in more than one building of the model plant. Critical zones are used to establish regions of explosion locations causing simultaneous multiple system failure, which have to be specially considered in the plant response analysis.

\section{Plant Response Analysis}

The plant response analysis is done using the Probabilistic Safety Assessment (PSA). PSA is a tool dealing with random events and its primary methods: fault tree analysis and event tree analysis are mostly used for assessment of systems reliability and NPP safety 10), 11), 15), 16). The models developed and analyzed are mostly very complex and approximations are commonly used to make analyses and to obtain results.

Models, analyses and results of PSA are considered for their use to contribute to the vulnerability analysis, because they represent a fairly consistent information about the safety of the complete NPP (it is essential that for vulnerability analyses based on PSA the fault trees and event trees are evaluated exactly) ${ }^{6), 24), 25)}$.

The models, analyses and results of PSA are considered in spite of some facts, which could prevent the use of existing PSA for vulnerability analyses:

- PSA is intended for use for random events, not for intentional events. 
- Many PSAs exist, where the fault trees and the event trees are not evaluated exactly (introduction of events with higher probability and rare events approximation are not compatible) ${ }^{6}$.

- Introduction of events, which are highly probable (and which are even not random events), may give the wrong results, because negligible portions truncated originally can become important contributions ${ }^{17)}$.

The plant response analysis is performed based on existing model of probabilistic safety assessment. Analysis is done by calculation of the core damage frequency (CDF) according to the plant damage analysis considering explosions of selected explosive masses at selected locations around the main plant buildings. The analysis is performed in a way that selected systems are evaluated in sense if they are operable or inoperable after an explosion of a selected mass of a selected explosive at a selected location. Systems that may be destroyed (and combinations of systems that may be destroyed) due to explosions are identified from screening of systems important for safety in a NPP. The analysis is performed for a variety of explosive masses and for all their possible locations considering three levels of the analysis, which are described in section II.5.

The results of calculations of core damage frequency are obtained in table form, where the selected components/systems and their combinations, which can be destroyed due to the selected extent of explosion at the selected location, are identified. Destroyed system means that the complete system is unavailable. Loss of a part of a system results in the loss of the system. PSA models are reevaluated for each table cell under conditions that the identified components/systems are unavailable. The modifications, which are made to the original PSA model for each table cell, include one or more changes of the following parameters:

- changes of initiating event frequencies,

- changes of unavailability of functional events (event tree headings),

- changes of unavailability of basic events,

- changes in common cause failure models and/or their respective parameters. 
But mostly, the modifications are made in sense that the equipment, which is considered unavailable due to the respective explosion, is changed from its original failure probability to failure probability of 1 .

The indication of the level of NPP vulnerability is indicated for each of identified systems failures and for all needed combinations of systems failures. Two states are identified as qualitative result based on quantitative and qualitative results of probabilistic safety assessment:

- very vulnerable state, where the qualitative results of PSA shows that at least one minimal cut set exists, which consist of events, which are all expected to occur under the assumed conditions and it therefore leads to a certain core damage (CD),

- vulnerable state, where the quantitative results of PSA based on sensitivity evaluations for determined combinations of assumed unavailable systems show significant increase of core damage frequency (CDF).

In the case of the analysis of a facility for which only a reliability assessment is performed instead of the full PSA, the described procedure is similar and it can be adopted for the reliability assessment, e.g.:

- The plant response analysis can be performed by assessment of facility failure probability instead of the core damage frequency (CDF).

- Facility failure can be considered instead of core damage.

- Qualitative and quantitative results of the fault tree analysis can be used instead of qualitative and quantitative results of the complete PSA.

\section{Consequences analysis}

The consequence analysis (as part of the overall method) represents a wide field, which includes and integrates several views:

- assessment of consequences of considered events (ground explosions) from physical and chemical point of view for the environment and public, directly and indirectly (short term and long term, respectively),

- assessment of consequences of considered events from the financial point of view, which can be important for the national economy. 
As the consequence analysis is a wide field, which includes and integrates several views (it can also only combine what has already been done), it is not further presented in this paper.

\section{Model of the Example Case}

The performance of the method is demonstrated on a full scale analysis of a typical PWR nuclear power plat ${ }^{6}$. For the purpose of this paper, its performance is demonstrated on a simplified example of a technological facility with a single building. Figure 1 shows the ground plan of the facility. The description of the facility and its building is the following:

- The building is of shape L.

- The building consists of 3 walls made of reinforced concrete with thickness $\mathrm{Dz}$, of 3 walls made of reinforced concrete with thickness Dz/2 and of two metal sheet walls with steel frame.

- The length of all walls equals to Db.

- The centre of the building is moved from the centre of the facility ground plan to the south for length $\mathrm{Ds}(\mathrm{Ds}=\mathrm{Db} / 2)$.

- The building is located in the facility area of dimensions Df*Df, which is surrounded by the fence of same dimensions (Df*Df); inside of the fence there is the controlled area.

It is assumed that the success criteria for the example technological facility require that at least one of the two systems (system 1 or system 2) is operable for the technological facility to be safely operable. Some common support subsystems (e.g. backup power source) are located in the building with system 2, so there is no total symmetry of both systems.

\section{Results}

The selected results for the simplified example of a technological facility with a single building are presented, although the methodology was tested also on a power plant. Only one figure of each result is shown in the paper, although results of subsections include many figures with variations of the amount and of the location of the explosive mass. 


\section{Results of Blast Analysis}

The results of the blast analysis include the identification of the explosive, the determination of the amount of explosive, and the determination of the corresponding blast parameters, which are used in the next steps. The equivalent amounts of TNT were selected for the analysis of bomb locations and varied to provide a sensitivity analysis.

\section{Results of Structural Response and Human Response to Blast Loading}

The load carrying capacity of the walls of different thicknesses was estimated in terms of the pressure impulse. The impulses causing maximum recoverable elastic deformation agree reasonably well with capacities assigned to the cylindrical reinforced concrete walls of explosive shelters ${ }^{26)}$. The distances for a given explosive mass and reflected pressure impulse have been derived, although they are not reported here, in order that the results cannot be used for malicious purposes.

The results show that explosion of the largest amount of explosive delivered by the largest truck with trailer (both full of explosive TNT) to the outside fence of the facility from direction of north and west:

- would not jeopardize systems inside the concrete building,

- would not injure the operators in the control room in the concrete building,

- would very likely cause substantial damage of steel frame buildings (if direction of explosion would be such that the steel frame buildings are not protected by concrete buildings),

- would very likely cause severe injuries of people and also casualties on various locations inside the fence.

Figure 2 shows the iso-damage contour lines around the detonation site marked by $\mathrm{x}$. The dashed thin and thick black circles denote the steel frame building "no damage" and "collapse" distances, the solid thin and thick black circles denote the "no damage" and "collapse" distances for reinforced concrete plate of selected thickness, and the dashed gray and solid gray circles denote the distances for eardrum rupture probability $50 \%$ and lung damage death probability $50 \%$. Figure 2 actually shows that if a selected bomb would explode at the location of the cross (in the centre of the circles on the figure), then: 
- a reinforced concrete wall of selected thickness would collapse if it is located inside the solid thick black circle, and would not be damaged if it is located outside the solid thin black circle,

- a steel frame building with metal sheet walls would collapse if it is located inside the dashed thick black circle, and would not be damaged if it is located outside the dashed thin black circle,

- a human being would probably become deaf if he or she is located inside the dashed gray circle (eardrum rupture probability $>50 \%$ ) and would probably die if he or she is located inside the solid gray circle (lung damage death probability $>50 \%$ ).

In addition, human response to blast loading is shown on figures on plant damage analysis in section IV.4.

\section{Results of Components and Systems Fragility Analysis}

Explosions outside of the fence (outside the controlled area) are generally found to be away enough that the walls of the building prevent the equipment of being damaged. So, only the bomb locations inside of the controlled area are presented. Features of system 1 and of system 2 are such that they are located in the middle of the building and the collapse of the wall does not destroy them automatically.

The results are commented within the section on results of plant damage analysis.

\section{Results of Plant Damage Analysis}

The results of the plant damage analysis are the most illustrative part of the vulnerability analysis of the example facility.

\section{(a) Bomb Location Outside of the Controlled Area}

The controlled area is defined as the location inside of the fence of the technological facility. Figure 3 shows the ground plan of the facility with a number of iso-damage contour lines. The iso-damage contour lines were determined based on the iso-damage contour circles around the bomb (Figure 2) for bomb locations, which were virtually moved along the fence. 
The results for a TNT truck bomb with a selected mass are shown. It was conservatively assumed that there are no obstacles (trees, soft ground), which would prevent to drive the truck with the bomb to the fence.

The dashed thick black line denotes the steel frame building "collapse" distance, the solid thin and thick black lines denote the "no damage" and "collapse" distances for reinforced concrete plate of a selected thickness, and the dashed gray and full gray lines denote the distances for eardrum rupture probability $50 \%$ and lung damage death probability $50 \%$. In the analysis it was conservatively assumed that the blast waves spread like in a free-air hemisphere, with no obstacles, which would weaken the blast.

Figure 3 shows the safe regions, where the damage is below a specified level for the bomb location outside the controlled area. If the reinforced concrete wall with selected thickness would be placed between the fence and solid thick black line, the wall would collapse. If the same wall would be placed more to the centre of the ground plan, as it is indicated by the solid thin black line, the wall would not be damaged. Similarly, if the steel frame buildings with metal sheet walls would be placed between the fence and the dashed thick black line, it would collapse. As there is no dashed thin black line, which would show the safe region for steel frame buildings with metal sheet walls, one can conclude that no safe region for such building exists in this example facility. If a person would stand between the fence and the solid gray line, he or she would probably die due to lung damage (lung damage death probability at the line is 50 $\%$ ). If a person would stand between the fence and the dashed gray line, he or she would probably become deaf (eardrum rupture probability at the line is $50 \%$ ). Figure 3 shows that none of the reinforced concrete walls would be damaged, but all metal sheet walls would be damaged and the metal sheet wall located on the east side of the building would probably also collapse. However, the systems inside the buildings would stay operational.

\section{(b) Bomb Location Inside of the Controlled Area}

The iso-damage contour lines for structural damage were determined using damage distances that were calculated according to the buildings properties. The reinforced concrete building with reinforced concrete walls (full and half thickness), and the steel frame building with metal sheet walls were considered (see example ground plan on Figure 1). 
The results for a TNT truck bomb with a selected mass, which equals to a $25 \%$ of the mass considered for the bomb locations outside of the controlled area, are shown. The reason for such selection lays in a fact, that as the location of the bomb is approaching closer to the target, the smaller explosive masses are becoming important enough to be considered.

Figure 4 shows the iso-damage contour lines for a selected TNT bomb placed inside the controlled area but outside of the technological buildings.

The dashed thin and thick black lines denote the steel frame building "no damage" and "collapse" distances and are plotted around the steel frame building. The solid thin and thick black lines denote the reinforced concrete building "no damage" and "collapse" distances for full and half thickness reinforced concrete plates, depending on the considered wall thickness, and are plotted around reinforced concrete buildings.

The iso-damage contour lines for human injuries were determined and plotted around the centre of the office. The dashed and solid gray lines denote the distances for eardrum rupture probability $50 \%$ and lung damage death probability $50 \%$ inside the office if the vehicle bomb would be at the location of the corresponding gray contour line. It was assumed that the blast parameters in the office are like they would be in a free-air hemisphere without any obstacles. This is probably much too conservative, since the office is located quite inside the building and a blast entering from a "free field" into branched channels rapidly looses its power ${ }^{18)}$. Therefore the gray human injury contour lines should be regarded only as very conservative bounds. They are presented mainly to give us an impression about human injury damage distances in free air, and therefore they are plotted also inside the buildings, where a vehicle bomb could not be located. It is however worth noting that injuries and casualties among the facility staff are likely to affect the performance of the plant operators (if the example facility would be a NPP).

In the present analysis the secondary and tertiary human injuries were not considered.

The iso-damage contour lines for system failure were determined according to the wall thickness of the concrete buildings inside which the systems are located, using the calculated damage distances. The iso-damage contour lines for system failure are denoted with the extra thick black lines, which are plotted near the concrete building 
walls. If the bomb would be located between the iso-damage contour lines for system failure and the building, the system inside the building would be destroyed.

The iso-damage contour lines were plotted in such a way that it is possible to establish to which part of the building they belong (the contour lines were not cut off at their intersections).

Figure 5 shows the critical zone for the explosion of a selected TNT bomb. If the bomb would be located in the critical zone, both systems would be destroyed simultaneously.

The typical size of the critical zone would be in the order of $1 \mathrm{~m}$ for a typical (possible) TNT load in a van.

The simplest solution to prevent the simultaneous destruction of both systems would be the placement of physical barriers around the critical zone in a way that it would be physically impossible to put the considered amount of explosive at this location.

\section{Results of Plant Response Analysis}

The results of the plant response analysis are a complement to the results of the plant damage analysis. Instead of PSA and sensitivity evaluations within PSA, only the reliability assessment using the fault tree was used for the simplified facility.

Table 1 and Figure 6 show the results for the simplified facility, where its reliability depends only on two systems and their subsystems.

Figure 6 shows vulnerability zones (GREY ... vulnerable; DARK GREY ... very vulnerable) for a selected bomb, which were identified in the second column of Table 1.

The vulnerability zones identified by the results of the reliability assessment are a complement to the critical zones determined by the plant damage analysis. Comparison and interpretation of the differences gives the standpoint for determining, which areas are to be physically protected.

The results indicate how much of territory should be physically protected, in the sense to prevent that a larger amount of explosive can be placed there. The proper physically protection could protect the plant systems and significantly reduce the vulnerability of the plant. 
As by showing the results of probabilistic safety assessment no wrong information can be revealed for malicious purposes, in continuation the results of probabilistic safety assessment for a three loop pressurized water reactor are shown, in addition.

Identification of systems that may be destroyed and identification of combinations of systems that may be destroyed due to explosions of selected explosive masses was based on screening of the important systems identified in PSA results. Table 2 shows selected components/systems and their combinations, which can be destroyed (i.e. complete system is unavailable; loss of a part of a system results in loss of a system) due to selected extent of explosion at selected location, and the results of the analysis, which give the indication of the level of NPP vulnerability (two states are stressed: very vulnerable state and vulnerable state).

As the loss of off-site power event is more vulnerable to an explosion (the switchyard is in open territory) than losses of other systems, which are inside the buildings, all identified components/system failures and their combinations were evaluated for the case of off-site power and for the case, when off-site power is lost. Identification of selected components/systems and their combinations was performed in connection with the physical locations of components/systems.

Probabilistic safety assessment models were reevaluated for each table cell under conditions that identified components/systems were unavailable.

The modifications, which were made to the original probabilistic safety assessment model NPP_S, mostly include changes of initiating event frequencies, changes of unavailability of functional events (event tree headings) and changes of unavailability of basic events. No detailed analysis was performed (e.g. human error probabilities probably increase, but were not analyzed at this point).

Table 2 contains all calculations of CDF for selected conditions, where selected systems or their combinations are destroyed due to an explosion of a selected amount of explosive mass at selected locations.

All calculations were performed in two ways indicated by [MCS EDIT] and by [MOD RUN]. The first way is a quick way of sensitivity analyses, where calculations are performed by changed unavailabilities of existing minimal cut sets based on results of the nominal PSA model. 
The second way is a more useful way of sensitivity analyses, where calculations are performed by new runs of adjusted PSA models (each change results in a complete reevaluation of a complete PSA model).

In Table 2 one can notice major differences of CDF results for the same conditions between both ways of CDF calculation: [MCS EDIT] and [MOD RUN]. The results obtained by [MOD RUN] were considered for determining vulnerability states, because [MOD RUN] results in complete reevaluation of the adjusted PSA model.

In Table 2 one can notice some very high CDF, which exceeds the value of $1 /$ ry. In spite of known differences between probabilities and frequencies, those values have to be dealt with great care due to the facts expressed in the beginning of section II.6.

High CDF values in this respect are not used as quantitative numbers. They are used only in sense that they give indication of conditions, which need more detailed care. In addition, it is important to distinguish:

Case, where CDF is high and qualitative analysis of probabilistic safety assessment shows that at least one minimal cut set exists, which consist of events, which are all expected to occur under the assumed conditions and it therefore leads to core damage $(\mathrm{CD})$ - very vulnerable state.

- $\quad$ Case, where CDF is high and quantitative results of probabilistic safety assessment based on sensitivity evaluations for determined combinations of assumed unavailable systems show significant increase of core damage frequency - vulnerable state.

Identification of both vulnerable states identified in Table 2 is further represented on the figures, which show vulnerability zones for different explosive masses (those figures are not presented here so that the results can not be used for malicious purposes). The figures indicate how much of territory is needed (if any) to fence in order to physically prevent that larger amount of explosive to be placed there to protect the plant systems in order to decrease the vulnerability of the plant.

\section{Discussion}

The methodology aiming at complex assessment of possible consequences following a deliberate damage in an operating nuclear power plant is further developed. 
The proposed method integrates phenomenological models of the cause of damage, material strength and injuries of human beings with the available NPP models used in probabilistic safety assessment. The main intention was to develop a quick and robust assessment method, which to a large extent relies on the already existing knowledge, models and analyses, which already exist. For these reasons, the existing framework of the external event analysis in PSA ${ }^{10)}$ seemed to provide an excellent starting point.

The difficulty of the approach is that it is faced with a potentially very large space of possible events, which may be considered for analysis. But on the other side, grouping of some events and gained experience with the consequences of those events decrease this difficulty.

Some of the considered events are connected with large uncertainties in damage predictions, e.g. component and system fragilities with respect to the full range of potential explosion effects. It is important to compare those predictions with other approaches ${ }^{25)}$ and in the case of the important contributors to risk to analyze them in more details.

The proposed method was successfully applied to an operating nuclear power plant. The particular damage source studied is an explosion of a device brought to the location by land transport. A broad sensitivity study varying the amount and position of the explosive has been conducted to assess the potential vulnerability of the plant and to set up the basis for the development of effective protection measures. For the obvious reasons, the illustrative example in the paper is limited to a simplified technological facility.

The results show qualitatively how the selected parameters of selected explosions change with the distance between the explosion location and the target. The effects of selected explosions to the reinforced concrete walls with selected thicknesses and to the metal sheet walls are shown. In addition, the effects of those explosions to a human being are assessed. The damage of systems that are placed inside the buildings is assessed. The combinations of destroyed equipment, which may cause facility failure, are identified. The locations, which may represent a potential threat if a selected amount of explosive is placed there, are identified graphically. These locations are the candidate 
locations for the areas needing effective protective measures such as for example placement of physical protection barriers.

The results show that explosion of the largest amount of explosive delivered by the largest truck with trailer (both full of explosive TNT) to the outside fence of the nuclear power plant:

- would not jeopardize safety systems inside the containment,

- would not injure the operators in the control room,

- would very likely cause substantial damage to steel frame buildings and to concrete buildings with thin concrete walls,

- would very likely cause loss of offsite power, but operators would have no difficulties to safely shutdown the plant,

- would very likely cause severe injuries of people (and also casualties) on various locations inside the fence (inside the controlled area).

The results show also the consequences of other amounts of explosive closer to the technological buildings. The results indicate that the facility could be effectively defended at relatively low costs (against the explosion of a device brought to the location by land transport). The placement of barriers, which hinder the approach of larger trucks proved to be a very efficient defense measure.

\section{Acknowledgement}

The Ministry of Higher Education, Science and Technology, Republic of Slovenia, supported this research (partly research program P2-0026, partly research project J2-6556 supported together with Slovenian Nuclear Safety Administration).

\section{References}

1) Stritar A., B. Mavko, J. Sušnik, B. Šarler, Some Aspects of Nuclear Power Plant Safety Under War Conditions, Nucl Technol, 1993; 101 (February): 193-201.

2) Čepin M, L. Cizelj, L. Fabjan, B. Mavko, Assessment of External Events (in Slovenian), IJS-DP-8648, IJS, Ljubljana, November 2002.

3) Garrick B. J, James E. Hall, Max Kilger, John C. McDonald, Tara O’Toole, Peter S. Probst, Elizabeth Rindskopf Parker, Robert Rosenthal, Alvin W. 
Trivelpiece, Lee A. Van Arsdale, Edwin L. Zebroski, Confronting the Risks of terrorism: Making the Right Decisions, Reliab Engng Syst Safety 2004; 86: $129-176$.

4) Aircraft Crash Impact Analyses Demonstrate Nuclear Power Plant's Structural Strength, NEI (information from EPRI report), December 2002.

5) Lyman E. S., The Vulnerability of Nuclear Power Plant Containment Buildings to Penetration by Aircraft, Nuclear Control Institute, Abridged Report from Full Report, 2001.

6) Čepin M., L. Cizelj, M. Leskovar, B. Mavko, Nuclear Power Plant Threat Analysis Report, Institut Jožef Stefan, IJS-DP-8905, 2004.

7) Cizelj L., M. Leskovar, M. Čepin, B. Mavko, A Method for Rapid Vulnerability Assessment of Structures Loaded by Outside Blasts, SMIRT, 2005.

8) Čepin M., L. Cizelj, M. Leskovar, B. Mavko, Vulnerability Analysis Considering Explosive Devices Delivered by Land Transport, PSA05, 2005.

9) Čepin M., Shutdown PSA and External Events PSA (in Slovenian), Training Course, Institut Jožef Stefan, 2002.

10) Probabilistic Risk Assessment Procedures Guide, NUREG/CR-2300, Vol. 1,2, US NRC, Washington DC, April 1982.

11) Čepin M., B. Mavko, A Dynamic Fault Tree, Reliab Engng Syst Safety 2002; 75 (1): 83-91.

12) Smith P.D., J.G.Hetherington, Blast and Ballistic Loading of Structures, Butterworth-Heineman Ltd, Oxford, Great Britain, 1994.

13) Mays G. C., P.D.Smith (eds.), Blast Effects on Buildings, Thomas Telford Publications, London, UK, 1995.

14) Kinney G. B., Explosive Shocks in Air, The MacMillan Company, New York, 1962.

15) Standard for Probabilistic Risk Assessment for Nuclear Power Plant Applications, ASME RA-S-2002, ASME, 2002.

16) PSA Applications Guide, Electric Power Research Institute, EPRI, TR-105396, 1995.

17) Čepin M., Analysis of Truncation Limit in Probabilistic Safety Assessment, Reliab Engng Syst Safety 2005; 87 (3):395-403. 
18) Bulson P. S., Explosive Loadings of Engineering Structures, E \& FN Pon, London, 1997.

19) Pilkey W. D., P.Y. Chang (eds.), Modern Formulas for Statics and Dynamics A Stress and Strain Approach, McGraw-Hill, 1978.

20) Harris C. M., C.E. Crede (eds.), Schock \& Vibration Handbook (2 ${ }^{\text {nd }}$ Ed.), McGraw-Hill, New York, 1976.

21) Gould P. L., Analysis of Shells and Plates, Springer Verlag, New York, 1988.

22) Brebbia C. A., V.Sánchez-Gálvez (eds.), Shock and Impact on Structures, Computational Mechanics Publications, Southampton, UK, 1994.

23) Rose T. A., P.D. Smith, Influence of the principal geometrical parameters of straight city streets on positive and negative phase blast wave impulses, International Journal of Impact Engineering 2002; 27: 359-376.

24) Peplow D. E., C. D. Sulfredge, R. L. Sanders, R. H. Morris, T. A. Hann, Calculating Nuclear Power Plant Vulnerability Using Integrated Geometry and Event Tree Models, Transactions of ANS 2002; 87: 559-561.

25) Peplow D. E., C. D. Sulfredge, R. L. Sanders, R. H. Morris, T. A. Hann, Calculating Nuclear Power Plant Vulnerability Using Integrated Geometry and Event Tree Models, Nuclear Science and Engineering 2004; 146: 71-87.

26) A.R.Farsoun, C.M.Baker, W.H. Zehrt Jr., Guide for Evaluating Blast Resistance of Nonstandard Magazines, U.S.Army Engineer Division, Huntsville, Al. 


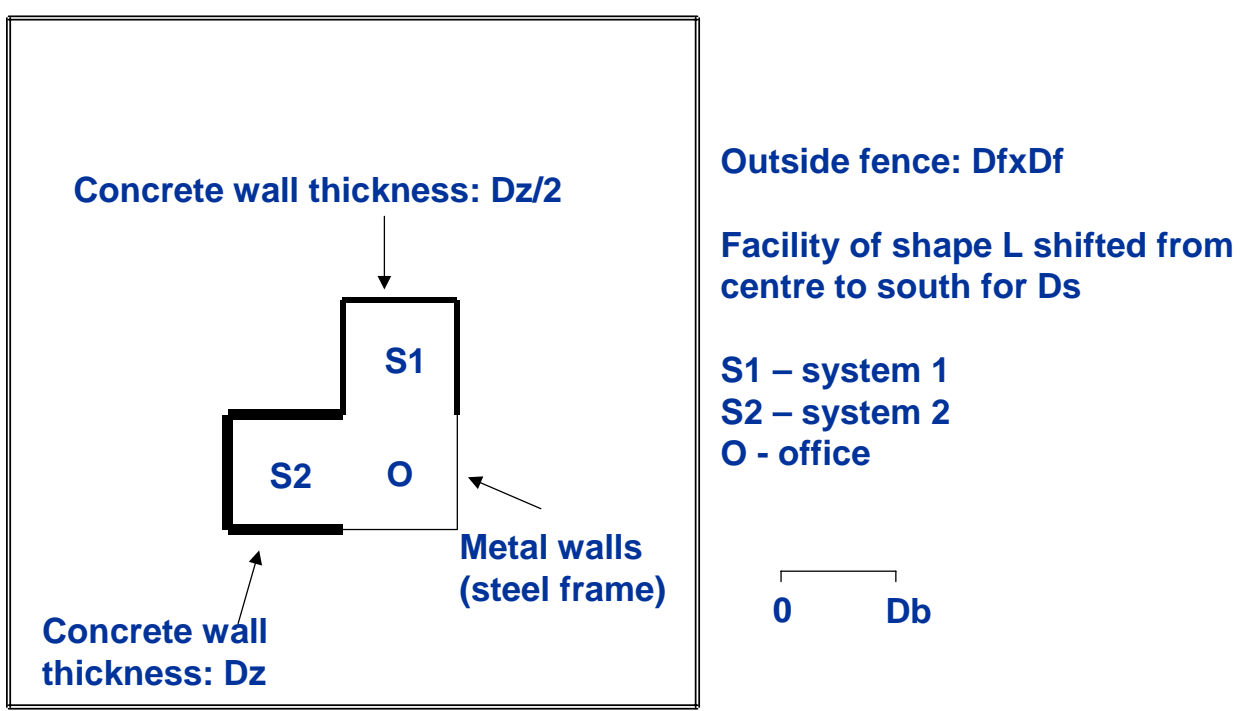

Figure 1: Ground Plan of the Example Facility with building. 


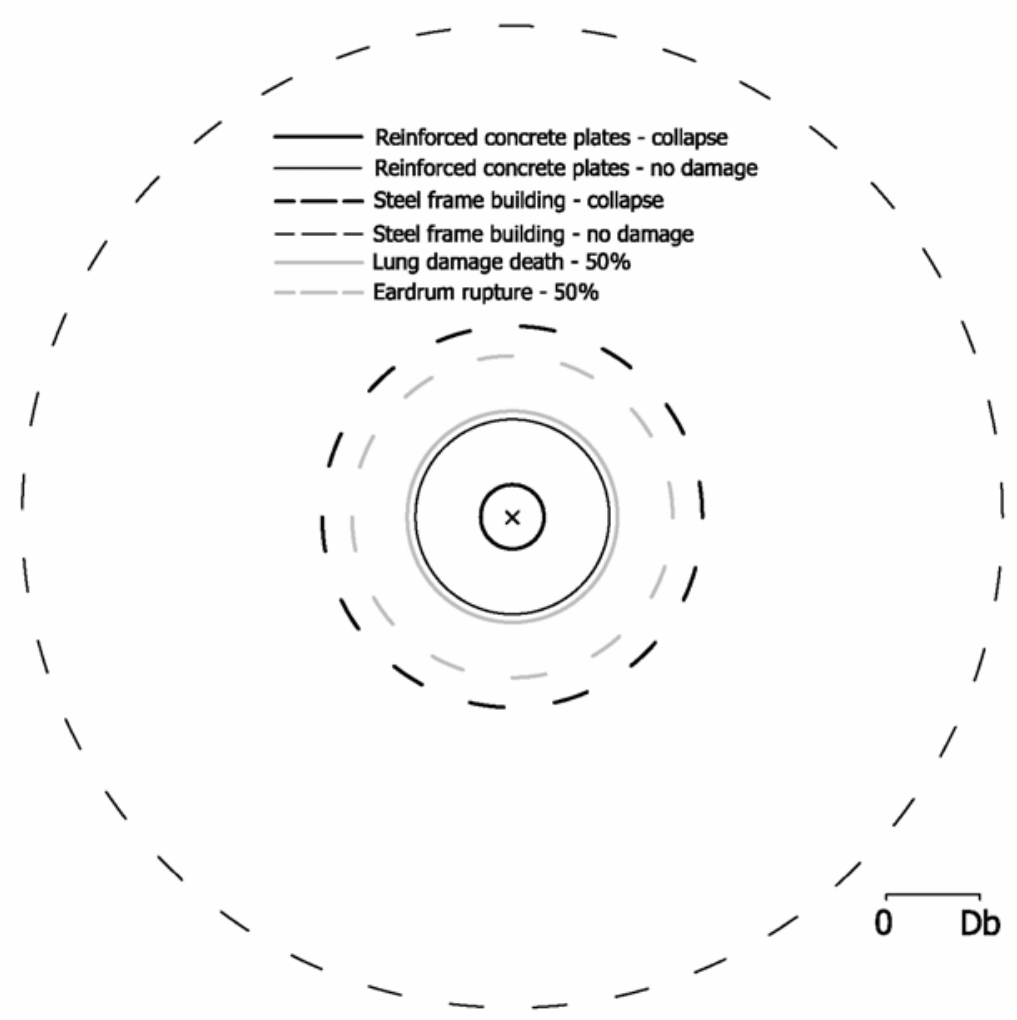

Figure 2: Iso-damage contour circles around a selected bomb. The distances are not to scale and may vary strongly with the variation of the explosive mass. 
Explosive mass: $X \mathrm{~kg}$ TNT

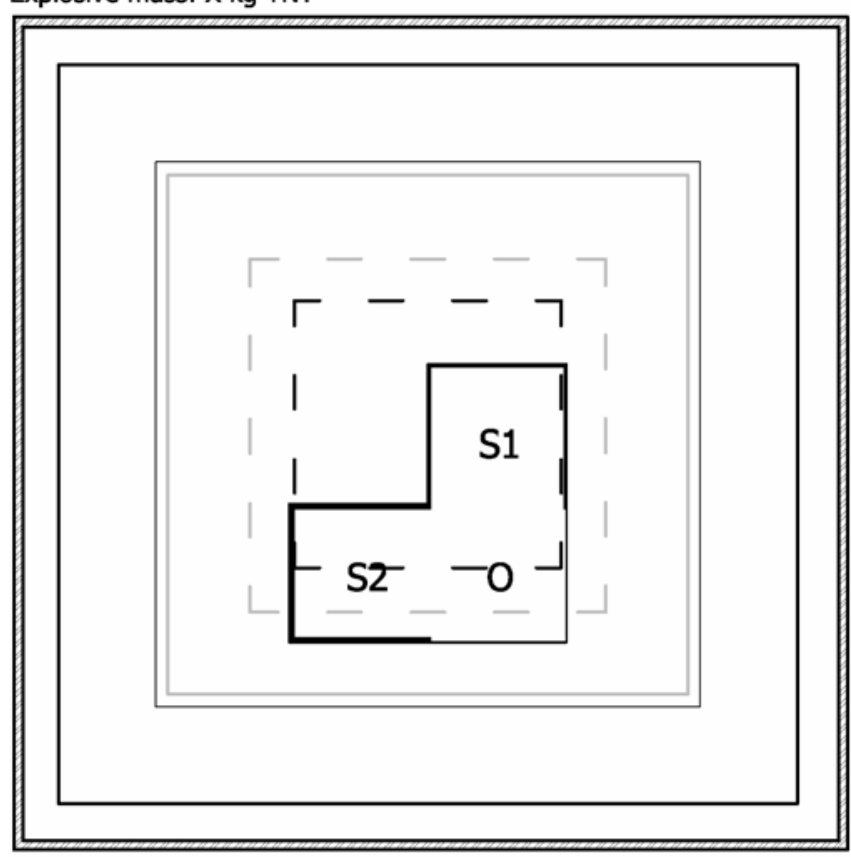

Reinforced concrete plates - collapse Reinforced concrete plates - no damage

- - - Steel frame building - collapse Lung damage death - $50 \%$ Eardrum rupture - $50 \%$

Figure 3: Safe regions for a selected TNT bomb at the border of the outside

fence. The distances are not to scale and may vary strongly with the variation of the explosive mass.

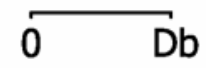




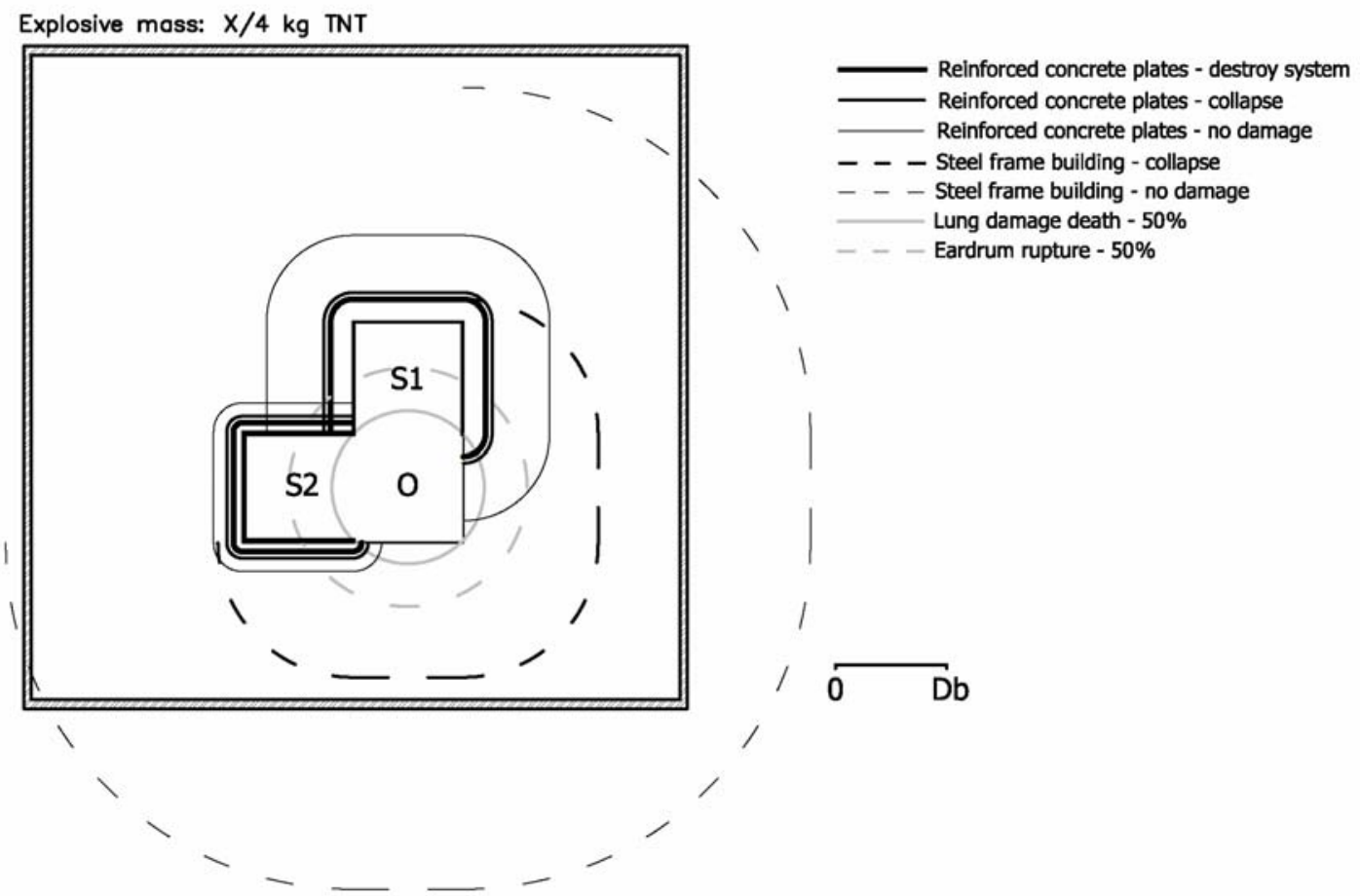

Figure 4: Iso-damage contour lines for a selected bomb inside the controlled area. The distances are not to scale and may vary strongly with the variation of the explosive mass. 


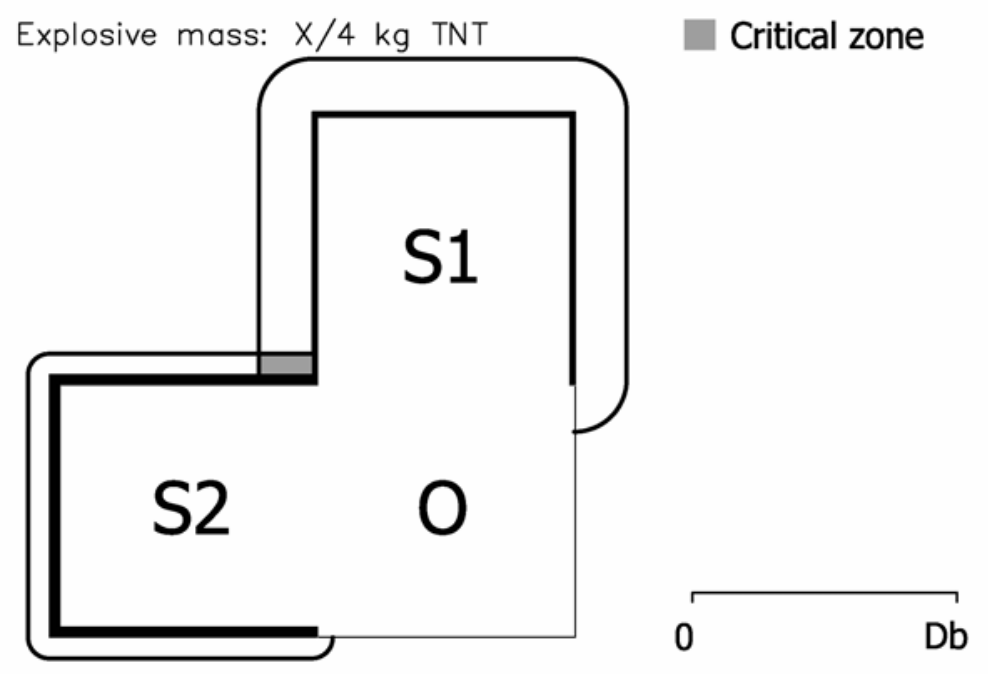

Figure 5: Critical zone for a selected bomb. The distances are not to scale and may vary strongly with the variation of the explosive mass. 


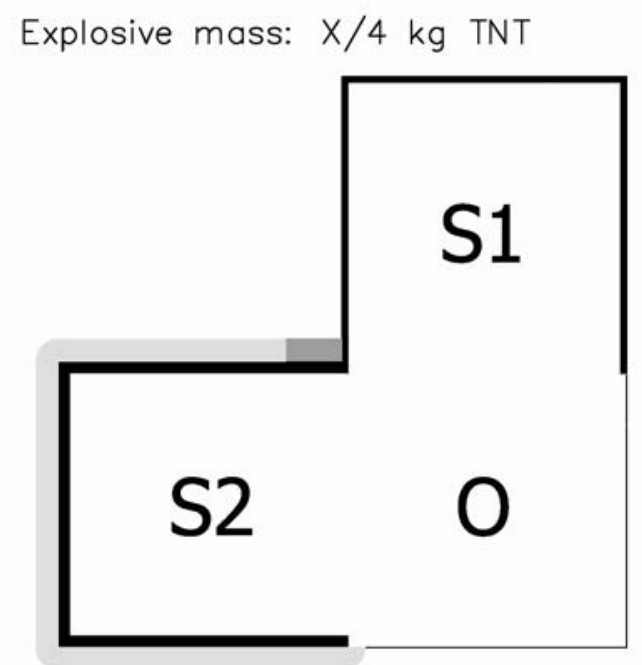

Vulnerable

Very vulnerable

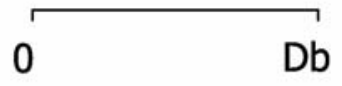

Figure 6: Vulnerability zones for a selected bomb. The distances are not to scale and may vary strongly with the variation of the explosive mass. 
Table 1: Assessment of facility failure probability under condition that certain systems are unavailable due to explosion.

\begin{tabular}{|l|l|}
\hline Identification of systems destroyed (i.e. unavailable) & Indication of facility vulnerability \\
\hline System 1 & \\
\hline System 2 (with subsystems in its building) & vulnerable* \\
\hline Systems 1 and 2 & very vulnerable** \\
\hline *vulnerable ... quantitative results of the fault tree analysis based on sensitivity evaluations \\
for determined combinations of assumed unavailable systems show a significant increase \\
of the facility failure probability \\
$* *$ very vulnerable... qualitative results of the fault tree analysis shows that at least one \\
minimal cut set (combination of component failures, which cause system failure) exists, \\
which consist of events, which are all expected to occur under the assumed conditions and \\
it therefore leads to facility failure \\
\hline GREY ... *vulnerable \\
\hline DARK GREY ...**very vulnerable
\end{tabular}


Table 2: Calculation of CDF under condition that certain systems are unavailable due to explosion

\begin{tabular}{|c|c|c|c|}
\hline $\begin{array}{l}\text { Identification of } \\
\text { equipment } \\
\text { destroyed (i.e. } \\
\text { unavailable) }\end{array}$ & $\begin{array}{l}\text { Method of } \\
\text { evaluation of } \\
\text { PSA model } \\
\text { changes }\end{array}$ & CDF (/ry) & $\begin{array}{l}\text { CDF (/ry) - } \\
\text { Loss of Off-site } \\
\text { Power }\end{array}$ \\
\hline System 1 & $\begin{array}{l}\text { [MCS EDIT] } \\
{[\mathrm{MOD} \text { RUN] }}\end{array}$ & $\begin{array}{l}1,6 \mathrm{E}-1 / \text { ry } \\
2,69 \mathrm{E}-2 / \text { ry }\end{array}$ & $\begin{array}{l}\text { 1,12/ry* } \\
1,95 \mathrm{E}-1 / \mathrm{ry} *\end{array}$ \\
\hline System 2 & $\begin{array}{l}\text { [MCS EDIT] } \\
{[\mathrm{MOD} \text { RUN] }}\end{array}$ & $\begin{array}{l}1,27 \mathrm{E}-4 / \mathrm{ry} \\
1,27 \mathrm{E}-4 / \mathrm{ry}\end{array}$ & $\begin{array}{l}8,15 \mathrm{E}-4 / \text { ry } \\
8,54 \mathrm{E}-4 / \mathrm{ry}\end{array}$ \\
\hline System 3 & $\begin{array}{l}\text { [MCS EDIT] } \\
{[\mathrm{MOD} \text { RUN] }}\end{array}$ & $\begin{array}{l}9,72 \mathrm{E}-3 / \mathrm{ry} \\
9,72 \mathrm{E}-3 / \mathrm{ry}\end{array}$ & $\begin{array}{l}1,02 \mathrm{E}-2 / \text { ry } \\
1,02 \mathrm{E}-2 / \text { ry }\end{array}$ \\
\hline System 4 & $\begin{array}{l}\text { [MCS EDIT] } \\
{[\mathrm{MOD} \text { RUN] }}\end{array}$ & $\begin{array}{l}\text { 6,59E-5/ry } \\
4,57 \mathrm{E}-5 / \mathrm{ry}\end{array}$ & $\begin{array}{l}3,86 \mathrm{E}-4 / \text { ry } \\
3,69 \mathrm{E}-4 / \text { ry }\end{array}$ \\
\hline System 5 & $\begin{array}{l}\text { [MCS EDIT] } \\
{[\mathrm{MOD} \text { RUN] }}\end{array}$ & $\begin{array}{l}9,55 \mathrm{E}-5 / \mathrm{ry} \\
5,45 \mathrm{E}-5 / \mathrm{ry}\end{array}$ & $\begin{array}{l}5,57 \mathrm{E}-1 / \text { ry * } \\
6,53 \mathrm{E}-2 / \text { ry * }\end{array}$ \\
\hline System 6 & $\begin{array}{l}\text { [MCS EDIT] } \\
{[\mathrm{MOD} \text { RUN] }}\end{array}$ & $\begin{array}{l}3,47 \mathrm{E}-5 / \text { ry } \\
3,47 \mathrm{E}-5 / \mathrm{ry}\end{array}$ & $\begin{array}{l}3,55 \mathrm{E}-4 / \text { ry } \\
3,59 \mathrm{E}-4 / \text { ry }\end{array}$ \\
\hline Systems 1 and 2 & $\begin{array}{l}\text { [MCS EDIT] } \\
{[\mathrm{MOD} \text { RUN] }}\end{array}$ & $\begin{array}{l}\text { 2,26/ry* } \\
3,22 \mathrm{E}-1 / \mathrm{ry} *\end{array}$ & $\begin{array}{l}\text { 3,21/ry* } \\
5,58 \mathrm{E}-1 / \mathrm{ry} *\end{array}$ \\
\hline $\begin{array}{l}\text { Systems 1,2 and } \\
3\end{array}$ & $\begin{array}{l}\text { [MCS EDIT] } \\
{[\mathrm{MOD} \text { RUN] }}\end{array}$ & $\begin{array}{l}2,26 / \text { ry } * * \mathrm{CD} \\
2,26 / \mathrm{ry} * * \mathrm{CD}\end{array}$ & $\begin{array}{l}3,22 / \text { ry } * * \mathrm{CD} \\
3,22 / \mathrm{ry} * * \mathrm{CD}\end{array}$ \\
\hline Systems 1 and 3 & $\begin{array}{l}\text { [MCS EDIT] } \\
\text { [MOD RUN] }\end{array}$ & $\begin{array}{l}\text { 1,71E-1/ry* } \\
1,52 \mathrm{E}-1 / \mathrm{ry} *\end{array}$ & $\begin{array}{l}1,16 / \text { ry } * * \mathrm{CD} \\
1,11 / \text { ry } * * \mathrm{CD}\end{array}$ \\
\hline Systems 2 and 3 & $\begin{array}{l}\text { [MCS EDIT] } \\
{[\mathrm{MOD} \text { RUN] }}\end{array}$ & $\begin{array}{l}1,03 \mathrm{E}-2 / \text { ry } \\
1,04 \mathrm{E}-2 / \text { ry }\end{array}$ & $\begin{array}{l}1,11 \mathrm{E}-2 / \text { ry } \\
1,13 \mathrm{E}-2 / \text { ry }\end{array}$ \\
\hline Systems 3 and 4 & $\begin{array}{l}\text { [MCS EDIT] } \\
{[\mathrm{MOD} \text { RUN] }}\end{array}$ & $\begin{array}{l}9,73 \mathrm{E}-3 / \text { ry } \\
9,72 \mathrm{E}-3 / \mathrm{ry}\end{array}$ & $\begin{array}{l}1,02 \mathrm{E}-2 / \text { ry } \\
1,02 \mathrm{E}-2 / \text { ry }\end{array}$ \\
\hline \multicolumn{4}{|c|}{$\begin{array}{l}\text { [MCS EDIT] ... calculations performed by changed unavailabilities of } \\
\text { existing minimal cut sets (results of nominal PSA model) } \\
\text { [MOD RUN] ... calculations performed by new runs of PSA models } \\
\text { (each change results in complete reevaluation of complete PSA model) }\end{array}$} \\
\hline \multicolumn{4}{|c|}{$\begin{array}{l}\text { GREY ... *vulnerable } \ldots \text { CDF }>0,05 / \text { ry (method of evaluation of PSA } \\
\text { model changes is [MOD RUN]) }\end{array}$} \\
\hline $\begin{array}{l}\text { DARK GREY ... } \\
\text { that a combinatior } \\
\text { damage }\end{array}$ & $\begin{array}{l}\text { ery vulnerab } \\
\text { minimal cut }\end{array}$ & $\begin{array}{l}\text {.CD ... qua } \\
\text { exists, whic }\end{array}$ & $\begin{array}{l}\text { ve analysis shows } \\
\text { ads to core }\end{array}$ \\
\hline
\end{tabular}


Figure 1: Ground Plan of the Example Facility with building.

Figure 2: Iso-damage contour circles around a selected bomb. The distances are not to scale and may vary strongly with the variation of the explosive mass.

Figure 3: Safe regions for a selected TNT bomb at the border of the outside fence. The distances are not to scale and may vary strongly with the variation of the explosive mass.

Figure 4: Iso-damage contour lines for a selected bomb inside the controlled area. The distances are not to scale and may vary strongly with the variation of the explosive mass.

Figure 5: Critical zone for a selected bomb. The distances are not to scale and may vary strongly with the variation of the explosive mass.

Figure 6: Vulnerability zones for a selected bomb. The distances are not to scale and may vary strongly with the variation of the explosive mass. 